\title{
Resistencia antihelmíntica en equinos. Observaciones sobre el período de reaparición de huevos luego del tratamiento con ivermectina en potrancas y yeguas madres
}

\author{
Caffe $\mathrm{G}^{1}$, Paz Bernard $\mathrm{B}^{1}$, Cerutti $\mathrm{J}^{1}$, Cooper $\mathrm{LG}^{1}$, Signorini $\mathrm{M}^{1,2}$, Anziani $\mathrm{OS}^{1,3^{*}}$ \\ 1 Universidad Católica de Córdoba, Argentina. \\ 2 Consejo Nacional de Investigaciones Científicas y Técnicas (CONICET), Argentina. \\ ${ }^{3}$ Instituto Nacional de Tecnología Agropecuaria (INTA), Estación Experimental Agropecuaria \\ Rafaela, Argentina. \\ * Correspondencia: OS Anziani. EEA INTA Rafaela. RN 34, km 227- Rafaela, Sata \\ Fe, Argentina. \\ E-mail: anziani.oscar@inta.gob.ar
}

Recibido: 27 Julio 2018. Aceptado: 19 Septiembre 2018. Disponible en línea: 20 Septiembre 2018

Editor: P. Beldomenico

RESUMEN. En los equinos parasitados por los pequeños estróngilos, un menor período requerido luego del tratamiento con antihelmínticos para la reaparición de huevos (PRH) de los nematodes en la materia fecal, puede ser utilizado como un indicador de la presencia de resistencia o pérdida de eficacia a estas drogas. En el presente trabajo se evaluó la eficacia clínica y el PRH luego de tratamientos con ivermectina $(0,2 \mathrm{mgr} / \mathrm{kg}$ ) en equinos adultos (yeguas madres) y jóvenes (potrancas) naturalmente parasitados por pequeños estróngilos en un establecimiento de la provincia de Tucumán. La eficacia clínica determinada en la segunda semana posttratamiento y utilizando un test de reducción en el conteo de huevos, osciló entre el 99,8 y el $100 \%$ en yeguas y potrancas respectivamente. Por su parte el PRH (considerado como el período post- tratamiento en que se alcanza el $10 \%$ del hpg pretratamiento o una reducción del mismo $<90 \%$ ) fue de seis y siete semanas para las potrancas y yeguas madres respectivamente $(p=0,001)$. En la sexta semana post- tratamiento, las potrancas mostraron poseer 11,74 veces más riesgo $($ IC $95 \%=2,58-53,38)$ de reaparición de huevos que las yeguas madres. Estas diferencias ocasionadas por la edad de los animales deberían considerarse cuando se realizan estudios para establecer el status de susceptibilidad o resistencia a los antihelmínticos basados en el PRH para evitar asunciones incorrectas sobre el mismo.

SUMMARY. Anthelmintic resistance in equines. Observations on the period of reappearance of eggs after treatment with ivermectin in fillies and broodmares. In horses parasitized by small strongyles, a shortened strongyle egg reappearance period (ERP) after treatment with anthelmintics is considered an early indicator of the presence of resistance or loss of efficacy to these drugs. In the present work, clinical efficacy and ERP were evaluated after treatments with ivermectin in adult horses (broodmares) and juveniles (its fillies) naturally parasitized by small strongyles in an farm of Tucumán province. The clinical efficacy determined in the second week after treatment, and using a test of reduction in the egg count, ranged between 99.8 and $100 \%$ in mares and fillies respectively. The ERP (considered as the post- treatment period in which $10 \%$ of the pre-treatment epg was reached or a reduction of it $<90 \%$ ) was six and seven weeks for the fillies and mother mares respectively $(p=0.001)$. In the sixth week after treatment, the fillies showed 11.74 times more risk $(95 \% \mathrm{Cl}=2.58-53.38)$ of reappearance of eggs than the brood mares. These differences caused by the age of the animals should be considered when conducting studies to establish the status of susceptibility or resistance to anthelmintics based on the ERP to avoid incorrect assumptions.

Palabras clave: pequeños estróngilos, equinos, ivermectina, resistencia

Key words: small strongyles, horses, ivermectina, resistance

\section{Introducción}

Los pequeños estróngilos (grupo Ciathostoma o Trichonema) son considerados desde una perspectiva global como los parásitos de mayor prevalencia en la especie equina (Brady y Nichols, 2009; Nielsen et al., 2016 ; Scott et al., 2015). Actualmente el control de estos nematodos se basa casi exclusivamente en la aplicación de antihel- 
mínticos y en este contexto, los bencimidazoles y las lactonas macrocíclicas aparecen como las drogas más utilizadas en la Argentina (Losinno et al., 2018). La resistencia de los nematodes a los bencimidazoles es un fenómeno común en nuestro país (Anziani y Catanzaritti, 2005; Cerutti et al., 2012) pero hasta el momento las lactonas macrocíclicas, como la ivermectina y moxidectina, mantienen una alta eficacia clínica bajo condiciones de campo (Anziani y Ardusso, 2017). El método más difundido en el mundo para determinar la eficacia de todos los tipos de antihelmínticos bajo condiciones de campo es un test in vivo conocido como test de reducción en el conteo de huevos (TRCH), el cual compara el número de huevos por gramo de heces (hpg) antes y 10 a 14 días después del tratamiento. Hasta el presente y en la especie equina, la documentación de la resistencia a las lactonas utilizando este test, han sido muy escasos. Sin embargo la eficacia reducida de ambas moléculas, medida por un acortamiento en el período de reaparición de huevos (PRH) luego del tratamiento, parece estar emergiendo en varios países del mundo (Lyons y Tolliver, 2013; Geurden et al., 2014; Matthews, 2014; Beasley et al., 2017). Así, el PRH es considerado actualmente como un alerta temprano al desarrollo de la resistencia a una droga determinada hasta que la eficacia reducida pueda ser confirmada con el TRCH (Nielsen et al., 2016). En el caso de los pequeños estróngilos y la ivermectina, el PRH cuando la droga fue introducida al mercado veterinario era de (al menos) 8 semanas, pero actualmente el mismo se ha reducido aproximadamente a 5 - 6 semanas (Nielsen y Reinemeyer, 2018) y estudios basados en necropsias indican que estas observaciones son el resultado de estadíos larvales luminales que sobreviven al tratamiento (Lyon et al., 2011; Lyons y Tolliver, 2013). La relación entre la edad de los animales y el PRH no ha sido dilucidada y hasta el momento las evidencias existentes son conflictivas. En este contexto, el objetivo del presente trabajo es comunicar las observaciones efectuadas sobre la eficacia y el PRH luego del tratamiento con ivermectina en equinos adultos y jóvenes del mismo sexo (yeguas madres y sus hijas) parasitadas naturalmente por pequeños estróngilos y mantenidas bajo las mismas condiciones de campo y manejo en un establecimiento de la provincia de Tucumán.

\section{Materiales y Métodos}

\section{a) Animales experimentales y lugar de la experiencia:}

Como animales experimentales se utilizaron 28 animales (14 yeguas y 14 potrancas) mayormente de biotipos deportivos (polo) pertenecientes a un centro de transplante embrionario de la provincia de Tucumán. La experiencia se desarrolló durante los meses de octubre a diciembre de 2017 y la edad de las yeguas osciló entre los 6 a los 17 años, mientras que la correspondiente a las potrancas fue de 9 a 12 meses. Ambas categorías habían permanecido juntas hasta aproximadamente los 60 días previos al inicio de la experiencia y luego permanecieron en un esquema de rotación sobre las mismas pasturas. El criterio para que las yeguas y sus crías sean incluidas en el estudio fue contar en el día 0 de la experiencia con un número de huevos de nematodes por gramo de heces (hpg) igual o superior a 200.

\section{b) Toma de muestras y técnicas coproparasitológicas:}

Las muestras de materia fecal se obtuvieron por masaje rectal o recogiendo las mismas directamente del suelo en los casos en que se observó defecar en forma espontánea a los animales experimentales. En el día 0 se tomaron muestras individuales pre-tratamiento de cada animal y luego del mismo se obtuvieron muestras semanales hasta la finalización de la experiencia. El hpg se determinó utilizando la técnica de Mc Master modificada (detección mínima $=10 \mathrm{hpg}$ ) y alícuotas de las mismas muestras de materia fecal fueron utilizadas para cultivos coproparasitológicos mantenidos en incubadoras a 22-25으 por 14 días. Las larvas obtenidas fueron recuperadas a través de un aparato de Baermann y clasificadas de acuerdo a Russell (1948).

\section{c) Tratamientos:}

Los animales fueron asignados a dos grupos de 14 animales cada uno y tratados en el día 0 de la experiencia en forma oral con 0,2 $\mathrm{mg} / \mathrm{kg}$ de ivermectina (Equvalan ${ }^{\circledR}$ pasta, Merial). El peso de las madres y sus crías se determinó en función de la longitud y el perímetro torácico de cada animal (Carol y Huntington, 1988) y para evitar cualquier posibilidad de sub dosificación se adicionó $10 \%$ al peso estimado.

\section{d) Determinaciones de eficacia y período de reaparición} de huevos:

Para determinar la eficacia clínica de la ivermectina, se utilizó el TRCH siguiendo el protocolo recomendado por la World Association for the Advancement of Veterinary Parasitology (Duncan et al., 2002) empleando la siguiente fórmula: $\mathbf{T R C H}=100 \times(1-T 2 / T 1)$ en donde T2 y T1 representan el promedio del hpg observado durante el post y pre- tratamiento respectivamente y considerando como eficacia para esta droga valores del TRCH > 95\% (Nielsen et al., 2016). El PRH se estableció como la semana post-tratamiento en la cual se observaron valores del TRCH $<90 \%$ lo cual representa el $10 \%$ del hpg pretratamiento (Nielsen et al., 2016; Beasley et al., 2016; Tzelos et al., 2017).En cada grupo la experiencia se dio por finalizada cuando se alcanzaron estos valores.

\section{e) Análisis estadísticos:}

Las diferencias en los valores del hpg de las potrancas y las yeguas al día del tratamiento con ivermectina fueron analizados por el test de Wilcoxon. Luego del tratamiento se utilizó la regresión lineal de Cox considerando a los valores del hpg como resultados y al peso y la edad de las yeguas madres y sus potrancas como factores confusores (programa Infostat, Universidad Nacional de Córdoba). 


\section{Resultados y discusión}

Los cultivos coprológicos pre-tratamiento (día 0 ) y posteriores al mismo (semana seis post-tratamiento) indicaron que el $100 \%$ de las larvas observadas en ambas categorías de equinos pertenecían a pequeños estróngilos. Estas observaciones eran esperables en función de los antecedentes del establecimiento que indicaban un uso mínimo de tres a cuatro aplicaciones anuales y masivas de antihelmínticos (mayormente ivermectina), lo cual implica generalmente la ausencia de grandes estróngilos cuando se aplican programas de control de esta naturaleza (Kaplan y Nielsen, 2010; Nielsen et al., 2016; Anziani y Ardusso, 2017). El promedio del hpg al momento del tratamiento en las yeguas madres y en sus potrancas fue de 592 (rango 230 -1.070) y 1.404 (rango $300-2.980$ ) respectivamente, mostrando diferencias significativas entre los valores de ambas categorías $(p=0,0033)$. En general y bajo condiciones de campo, es un hecho común que los valores del hpg en animales jóvenes resulten significativamente mayores (probablemente por una menor inmunidad relativa) en relación a los adultos (Herd y Gabel, 1990; Chapman et al., 2003; Nielsen et al., 2016) y esta situación también fue observada en el presente estudio. En el TRCH llevado a cabo en la segunda semana luego de la administración de ivermectina, la eficacia de la droga fue de $99,9 \%$ en las yeguas y $100 \%$ en las potrancas, la cual concuerda con observaciones previas desarrolladas en nuestro país en equinos del área central de la Argentina (Anziani y Ardusso, 2017). Por su parte, el $\mathrm{PRH}$ (período post- tratamiento hasta alcanzar TRCH < $90 \%)$ resultó de seis semanas en las potrancas y de siete en las yeguas madres $(p=0,001)$. El peso inicial de los animales no pareció influir sobre el PRH y a la sexta semana post - tratamiento, las potrancas mostraron poseer 11.74 veces más riesgo (IC $95 \%=2,58-53,38$ ) de reaparición de huevos que las yeguas madres. Una síntesis de estos resultados son presentados en la Tabla 1.

Las investigaciones de campo sobre la actividad de las drogas antihelmínticas utilizando el TRCH y el PRH adolecen de limitantes, como la dificultad para incluir un número importante de animales en los tratamientos, la naturaleza sobre-dispersa de las poblaciones de nematodes, así como la baja sensibilidad de las técnicas disponibles para las determinaciones del hpg (Lester et al., 2013). Las poblaciones parasitarias también difieren en función de los antecedentes previos de pastoreo, la edad de los hospedadores e incluso la raza y el sexo de los equinos (Komas et al., 2010; Relf et al., 2013 ; Nielsen y Lyons, 2017) y esta variabilidad hace difícil comparar los resultados entre múltiples estudios. En rumiantes y cerdos, la farmacocinética observada en la administración parenteral (intramuscular y subcutánea) o por derrame dorsal (pour on) de la ivermectina muestra variaciones individuales atribuidas a la condición corporal y/o a diferencias en el metabolismo y eliminación de la droga en relación a la edad, sexo o estado fisiológico de los animales (Scott y McKellar, 1992 ; McKellar y Benchaoui, 1996 ; Gokbulut et al., 2015) En el caso de los equinos, pero tratados en forma oral con ivermectina, estudios recientes muestran que el estado nutricional no parecen afectar significativamente los parámetros farmacocinéticos de la droga (Sokol et al., 2015) pero se desconoce si los mismos pueden ser modificados en función de la edad de los animales.

Un menor PRH luego del tratamiento con ivermectina en animales jóvenes comparado con adultos, fueron informados en E.E.U.U. (Herd y Gabel, 1990) mientras la ausencia de diferencias entre categorías fueron publicadas en Ho-landa y Australia (Boersema et al., 1996; Bealsey et al., 2017). Sin embargo, las comparaciones con estos trabajos son difíciles de establecer con la presente experiencia ya que en los dos primeros, el PRH se estimó en forma diferente al nuestro, tomando como tal al período post-tratamiento durante el cual el promedio del hpg no excedió el valor de 100. En nuestra experiencia se adoptó el criterio sugerido por la American Association of Equine Practitioners (Nielsen et al., 2016) la cual define al PRH (y para las lactonas macrocíclicas) como la semana post-tratamiento en la cual el TRCH es $<$ al $90 \%$ ( $\geq 10 \%$ del hpg al momento del tratamiento). Esta última definición es la más utilizada actualmente y es considerada como una estimación más conservadora y confiable de la sensibilidad de un antihelmíntico a nivel poblacional (Mattheus, 2014). En el tercer trabajo (Bealsey et al., 2017) el PRH se estimó en igual forma y con similar número de animales, pero estos pertenecían a tres establecimientos distintos y ninguno de ellos contaba con ambas categorías en el mismo predio, lo cual es reconocido por los mismos autores como una limitante para evaluar la hipótesis de la edad y su relación con el PRH.

Tabla 1. Eficacia comparativa en el TRCH luego de la administración oral de ivermectina en yeguas madres y sus potrancas y período de reaparición de huevos en cada grupo. Letras diferentes en la misma columna difieren estadísticamente (a y b $p=0.030 ; c$ y d $p=0.001$ ). $\mathrm{NC}=$ no corresponde
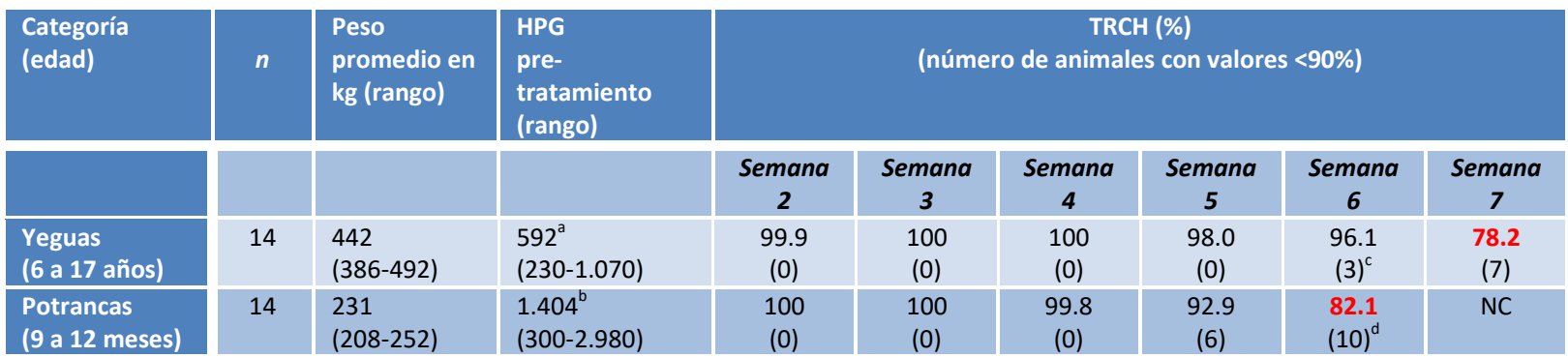

\begin{tabular}{|l|l|l|}
\hline & & \\
\hline 14 & 442 & $592^{\mathrm{a}}$ \\
& $(386-492)$ & $(230-1.070)$ \\
\hline 14 & 231 & $1.404^{\mathrm{b}}$ \\
& $(208-252)$ & $(300-2.980)$ \\
\hline
\end{tabular}

\begin{tabular}{|c|c|c|c|c|c|}
\hline Semana & Semana & Semana & Semana & Semana & Semana \\
\hline $\mathbf{2}$ & $\mathbf{3}$ & $\mathbf{4}$ & $\mathbf{5}$ & $\mathbf{6}$ & $\mathbf{7}$ \\
\hline 99.9 & 100 & 100 & 98.0 & 96.1 & 78.2 \\
\hline$(0)$ & $(0)$ & $(0)$ & $(0)$ & $(3)^{\mathrm{c}}$ & $(7)$ \\
\hline 100 & 100 & 99.8 & 92.9 & 82.1 & $\mathrm{NC}$ \\
$(0)$ & $(0)$ & $(0)$ & $(6)$ & $(10)^{\mathrm{d}}$ & \\
\hline
\end{tabular}


El número de animales presentados en este estudio (14 yeguas y 14 potrancas) fue relativamente bajo, pero consideramos que nuestros datos y conclusiones son particularmente relevantes debido al mismo biotipo, sexo y antecedentes comunes de los animales experimentales. En este contexto, todas las potrancas fueron nacidas en el mismo establecimiento donde las madres fueron preñadas, mantuvieron su gestación y fueron expuestas e infectadas con parásitos intestinales a partir de las mismas pasturas sobre las que pastorearon. A conocimiento de los autores, este sería el primer estudio sobre el PRH en animales jóvenes vs adultos tratados con ivermectina que considera estas variables y que se realiza en un mismo establecimiento.

En síntesis, actualmente el PRH es utilizado como una herramienta para detectar evidencias de la disminución de eficacia o indicador temprano de la resistencia de los pequeños estróngilos a los antihelmínticos, pero la mayoría de los estudios han sido realizados sin considerar muchas veces los biotipos, actividad de los animales, antecedentes de pastoreo, género, sexo ni edades de los mismos y existe la posibilidad de que estas variables puedan haber influenciado los resultados. También podrían existir diferencias farmacocinéticas de la ivermectina entre categorías. En nuestro estudio el número de los animales experimentales limita la extrapolación general de los resultados pero las diferencias encontradas entre potrancas y sus yeguas madres dentro de un mismo establecimiento, enfatizan la necesidad de estudios adicionales al respecto. Mientras esta información no esté disponible ni exista un protocolo estandarizado para este test in vivo, un cuidado especial debería darse a la homogeneidad en la edad de los animales involucrados en determinaciones del PRH a fines de evitar asunciones que pueden resultar incorrectas.

\section{Agradecimientos}

A la secretaría de Investigación de la Universidad Católica de Córdoba y a la Cooperadora de la EEA INTA Rafaela por la financiación del presente trabajo.

\section{Bibliografía}

Anziani OS, Catanzaritti H. 2005. Resistencia a los benzimidazoles en nematodes de los equinos en Santa Fe, Argentina. Vet Arg. 218: 571-578.

Anziani O, Ardusso G. 2017. Resistencia a los antihelmínticos en nematodes intestinales que parasitan a los equinos en la Argentina. RIA (INTA) 43: 1-12.

Beasley A, Kotze A, Allen K, Coleman G. 2017. A survey of macrocyclic lactone efficacy in Australian Cyathostomin populations. Vet. Parasitol. Reg. Stu. Rep. 8: 127-132

Boersema JH, Eysker M, Maas J, WM van der Aar. 1996 Comparison of the reappearance of strongyle eggs in foals, yearlings, and adult horse after treatment with ivermectin or pyrantel. Vet Quarterly 8:7-9.

Brady H, Nichols W. 2009. Drug resistance in equine parasites: an emerging global problem. J. Equine Vet. Sci. 29: 285-295.

Carroll CL, Huntington PJ. 1988. Body condition scoring and weight estimation of horses. Equine Vet. J. 20:41-45.

Cerutti J, Cooper L, Caffe G, Cervilla N, Muchiut S, Anziani OS. 2012. Resistencia de los pequeños estrongilidos (grupo Ciatostoma) a los bencimidazoles en equinos del área central de la Argentina. Rev. InVet. 14: 41-46.

Chapman MR, French DD, Klei TR. 2003. Prevalence of strongyle nematodes in naturally infected ponies of different ages and during different seasons of the year in Louisiana. J. Parasitol. 89: 309-314.

Duncan J, Abbott E, Arundel J, Eysker M, Klei TR, Krecek RC, Lyons ET, Reinemeyer C, Slocombe J. 2002. World Association for the Advancement of Veterinary Parasitology (WAAVP): second edition of guidelines for evaluating the efficacy of equine anthelmintics. Vet. Parasitol. 103:1-18.

Geurden T, van Doorn D, Claerebout E, Kooyman F, De Keersmaecker S, Vercruysse J, Besognet B, Vanimisetti B, di Regalbono AF, Beraldo P, Di Cesare A, Traversa D. 2014. Decreased strongyle egg re-appearance period after treatment with ivermectin and moxidectin in horses in Belgium, Italy and The Netherlands. Vet. Parasitol. 204: 291-296.

Gokbulut C, Cirak VY, Senlik B, Aksit D, McKellar QA. 2111. The effects of different ages and dosages on the plasma disposition and hair concentration profile of ivermectin following pour-on administration in goats. J. Vet. Pharmacol. Ther. 34: 70-75.

Herd RP, Gabel AA. 1990. Reduced efficacy of anthelmintics in young compared with adult horses. Equine Vet. J. 22: 164-169.

Kaplan RM, Nielsen MK. 2010. An evidence-based approach to equine parasite control: It ain't the 60 s anymore. Equine Vet. Ed. 22: $306-313$

Komas S, Cabaret J, Skalska M, Nowosad B. 2010. Horse infection with intestinal helminths in relation to age, sex, access to grass and farm system. Vet. Parasitol. 174: 285-29.

Lester HE, Spantonb J, Stratfordf CH, Bartleya DJ, Morganc ER, Hodgkinsond JE, Coumbee K, Maire T, Swane B, Lemone G, Cooksonf R, Matthewsa JB. 2013. Anthelmintic efficacy against cyathostomins in horses in Southern England. Vet. Parasitol. 197: 189-196.

Losinno SJ, Aguilar J, Carbonetti L, Ferniot E, San Esteban F, Flores Suares CM. 2018. A survey on parasite control in sport horses of Argentina and other regional countries. Vet. Parasitol. Reg. Stu. Rep. 13: 74-78.

Lyons ET, Tolliver SC, Collins SS. 2011. Field tests demostrating reduced activity of ivermectin and moxidectin against small strongyles in horses on 14 farms in Central Kentucky in 20072009. Parasitol. Res. 108: 355-360.

Lyons ET, Tolliver SC. 2013. Further indication of lowered activity of ivermectin on immature small strongyles in the intestinal lumen of horses on a farm in Central Kentucky. Parasitol. Res. 12: 889-891. 
Matthews JB. 2014. Anthelmintic resistance in equine nematodes. Int. J. Parasitol. Drugs Drug Res. 4: 310-315.

McKellar QA, Benchaoui HA. 1996. Avermectins and milbemycins. Vet. Pharmacol. Ther. 19: 331-51.

Nielsen MK, Mittel L, Grice A, Erskine M, Graves E, Vaala W, Tully R, French D, Bowman R, Kaplan R. 2016. AAEP Parasite Control Guidelines. American Association of Equine Practitioners. Available online at www.aaep.org

Nielsen MK, Lyons E T. 2017. Encysted cyathostomin larvae in foals - progression of stages and the effect of seasonality. Vet. Parasitol. 236: 108-112.

Nielsen MK, Reinemeyer CR. 2018. Case 17: Ivermectin Egg Reappearance. In: Nielsen MK, Reinemeyer CR (Eds.) Handbook of Equine Parasite Control, 2nd Ed.. Wiley-Blackwell. 284pp.

Relf VE, Morgan ER, Hodgkinson JE, Mathews JB.2013. Helminth egg excretion with regard to age, gender and management practices on UK Thoroughbred studs. Parasitology 140: 641-652.

Russell AF. 1948. The development of helminthiasis in Thoroughbred foals. J. Comp. Path. Therap. 58:107-127.

Scott EW, McKellar QA. 1992. The distribution and some pharmacokinetic parameters of ivermectin in pigs. Vet. Res. Comm. 16: 139-46.

Scott I, Bishop RM, Pomroy WE. 2015. Anthelmintic resistance in equine helminth parasites - a growing issue for horse owners and veterinarians in New Zealand? N. Z. Vet. J. 63: 188-198.

Sokół R, M. Raś-Noryńska M, Michalczyk M, Jasiecka A, Ziółkowsk $H$, Jaroszewski. 2015. A comparison of the efficacy and pharmacokinetics of ivermectin after spring and autumn treatments against Cyathostominae in horses. Polish J. Vet. Sci. 18: $371-377$.

Tzelos T, Barbeito JSG, Nielsen MK, Morgan ER, Hodgkinson JE, Matthews JB. 2017. Strongyle egg reappearance period after moxidectin treatment and its relationship with management factors in UK equine populations. Vet. Parasitol. 237: 70-76. 